\title{
Opposite Effects of A Singlet Oxygen Quencher on Photochemical Degradation of Dicyano-Substituted Poly(phenylenevinylenes) with Different Side Chains
}

\author{
Logan P. Sanow and Cheng Zhang* \\ Department of Chemistry and Biochemistry, South Dakota State University, Brookings, SD 57006 \\ Correspondence to: Cheng Zhang (E-mail: cheng.zhang@sdstate.edu)
}

\begin{abstract}
RO-diCN-PPV and C8-diCN-PPV, poly(1,4-phenylene-1,2-dicyanovinylene) with alkoxy and octyl side chains, have recently been shown to photodegrade via a singlet oxygen mechanism, and RO-diCNPPV is seven times more stable. To improve photostability, 1,4-diazabicyclo[2.2.2]octane (DABCO), a singlet oxygen quencher, was used as a dopant. To our surprise, DABCO exhibited opposite effects on their photodegradation. With 15 mol\% DABCO, degradation rate of C8-diCN-PPV decreased by 65\%, while that of RO-diCN-PPV increased by $246 \%$. The DABCO content in C8-diCN-PPV film remained unchanged during 20 minutes of illumination, but mostly disappeared in RO-diCN-PPV in only 5 minutes due to decomposition. IR and MW analysis results suggest that DABCO slowed down degradation of C8-diCN-PPV without altering the mechanism, but accelerated RO-diCN-PPV photodegradation by initiating a radical process. C8-diCN-PPV's HOMO energy is lower than that of $\mathrm{DABCO}$ by $1.78 \mathrm{eV}$, a gap too wide for efficient electron transfer to happen. On the other hand, the HOMO of RO-diCN-PPV is only lower by $1.14 \mathrm{eV}$, allowing DABCO to donate electron to photoexcited RO-diCN-PPV to initiate a radical process that damaged the polymer and destroyed DABCO itself. It was also found that, in RO-diCN-PPV, radical decomposition takes very different paths from those of RO-PPVs and produce very different products.
\end{abstract}

KEYWORDS: Photodegradation, Photostability, Dicyano poly(phenylenevinylene), singlet oxygen, superoxide radical, DABCO

\section{INTRODUCTION}

ח-Conjugated organic materials have many (potential) applications such as photovoltaics, light emitting diodes, solid-state dye lasing, biological imaging and sensing, and chemical sensing. However, photochemical stability is a major concern for these materials. ${ }^{1-5}$ For electron-rich polymers, such as poly(3hexylthiophenes) and poly(p-phenylenevinylenes) (PPV), a radical degradation mechanism has been established..$^{6-9}$ When illuminated in the presence of oxygen, the polymer donates an electron through charge transfer to an oxygen molecule to form a polymer cation and a superoxide radical anion. In the case of PPVs, the superoxide radical anion can abstract a hydrogen from a side chain to form a carbon radical. ${ }^{8}$ It can also attack a vinylene unit in the backbone to produce a peroxide anion and a carbon radical. ${ }^{8}$ Hoke et al. and Dam et al. have shown that the LUMO (lowest unoccupied molecular orbital) energy of a polymer is directly related to the rate of 
photodegradation. ${ }^{10,11}$ In our earlier reports, poly[2,5-bis(decyloxy)-1,4-phenylene-1,2-

dicyanovinylene] (RO-diCN-PPV), and poly(2,5dioctyl-1,4-phenylene-1,2-dicyanovinylene) (C8diCN-PPV) (Figure 1$)^{12,13}$ were shown to have photostability up to 250 times that of RO-PPV, due to reduced LUMO energy, $-3.54 \mathrm{eV}$ and $3.69 \mathrm{eV}$ as compared to $-2.91 \mathrm{eV}$ of RO-PPV. Superoxide radical generation was shut down in these two polymers, ${ }^{13}$ however, singlet oxygen mechanism, though less aggressive, still limited their photostability, and C8-diCN-PPV degraded seven times faster due to faster ${ }^{1} \mathrm{O}_{2}$ generation in the polymer.

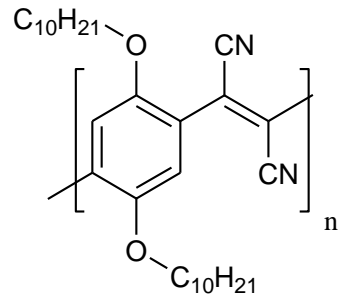

RO-diCN-PPV

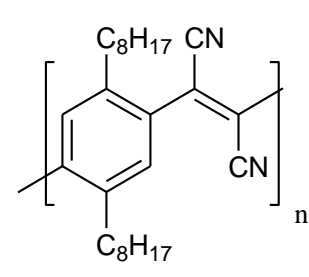

C8-diCN-PPV
Figure 1. Chemical structures of two diCN-PPV polymers.

For organic materials that undergo photodegradation via radical oxidation mechanisms, radical scavengers can be used to slow down the processes. ${ }^{14,15}$ For materials that are sensitive to singlet oxygen, ${ }^{1} \mathrm{O}_{2}$ quenchers such as 1,4-diazabicyclo[2.2.2] octane (DABCO) $)^{16}$ and other tertiary amines ${ }^{17}$ are effective antioxidants and can be used to improve the lifetime of dyes in dye lasers and fluorescence microscopy, ${ }^{18}$ as they can physically remove the excitation energy from ${ }^{1} \mathrm{O}_{2}$ without undergoing permanent change themselves and convert the energy to heat. ${ }^{19}$

In this work, the effects of DABCO on the photostability of RO-diCN-PPV and C8-diCN-PPV were investigated. To our surprise, the singlet oxygen quencher affected photodegradation of the two similar polymers in opposite ways, a situation not have been found in the literature. The differences in the mechanisms are elucidated with the help of UV-vis absorption and infrared spectroscopic methods, and molecular weight analysis.

\section{EXPERIMENTAL}

The polymer films were spin-coated from polymer solutions in o-dichlorobenzene on glass substrates for UV-vis measurements and drop casted on $\mathrm{KBr}$ plates for IR measurements. The optical density of the films on glass substrate was kept between 0.2 and 0.3 , except when shown otherwise. Films on $\mathrm{KBr}$ plates are about 10 times thicker as polymer absorption is about one order of magnitude weaker in the infrared $\left(4000-650 \mathrm{~cm}^{-1}\right)$ than in the visible region.

A high power Newport light source (model 66903) equipped with a $300 \mathrm{~W}$ xenon arc lamp and a Newport power supply (Model 69911) was used for the photostability study. The xenon lamp produces light of broad spectrum from $200 \mathrm{~nm}$ to IR. The unfiltered output was focused on sample to generate an intensity of $2200 \mathrm{~mW} / \mathrm{cm}^{2}, 22$ times the intensity of one standard sun. UV-vis or IR analyses were performed immediately after illumination.

UV-vis spectra were obtained from an Agilent 8453 photodiode array UV-vis absorption spectrophotometer. Infrared spectra were obtained on a PerkinElmer Spectrum Two FT-IR spectrometer with a DynaScan interferometer, $\mathrm{KBr}$ optics and a temperature-stabilized DTGS detector. Molecular weights were determined using an Agilent 1100 series HPLC system, equipped with a diode array detector and two GPC columns (PL1110-6500, PLGel $5 \mathrm{~mm}$ MIXED-C $7.5 \times 300 \mathrm{~mm}$ ). Polystyrenes were used as the standards for conventional calibration and tetrahydrofuran as the solvent.

\section{RESULTS AND DISCUSSION}

\section{Photostability}

Photooxidation of the polymer film samples was monitored using UV-vis absorbance (Figure 2 and Figure 3). Since the light intensity and oxygen concentration are constant, the 
photodegradation follows the first order rate law, ${ }^{13}$

$$
\log \frac{A}{A_{0}}=-K t
$$

where $K$ is the effective polymer photodegradation constant, $A_{0}$ is the initial peak absorbance, and $A / A_{0}$ is the normalized peak absorbance. $\log \left(A / A_{0}\right)$ is plotted as a function of time in Figure 4. The absolute values of the slopes are the effective degradation constants. Since decomposition product can have absorption tailing into the peak region of the polymer and causes the data points to deviate from a linear dependence as the illumination continues. This effect is much more significant in the pristine C8-diCN-PPV film than other samples. Therefore, in Fig. 4, only the data points in the first 5 minutes are used to calculate the slope for C8-diCN-PPV. The initial photooxidation rate of C8-diCN-PPV is 7 times greater than that of RO-diCN-PPV.

With 15 mol\% DABCO doping, the degradation rate of C8-diCN-PPV film decreased by $65 \%$ (Figure 2 and Figure 4), in consistency with the singlet oxygen mechanism. The effect of DABCO in RO-diCN-PPV may not appear significant when comparing the top two charts of Figure 3. After the spectra are magnified (Figure 3 bottom), it becomes clear that, with DABCO

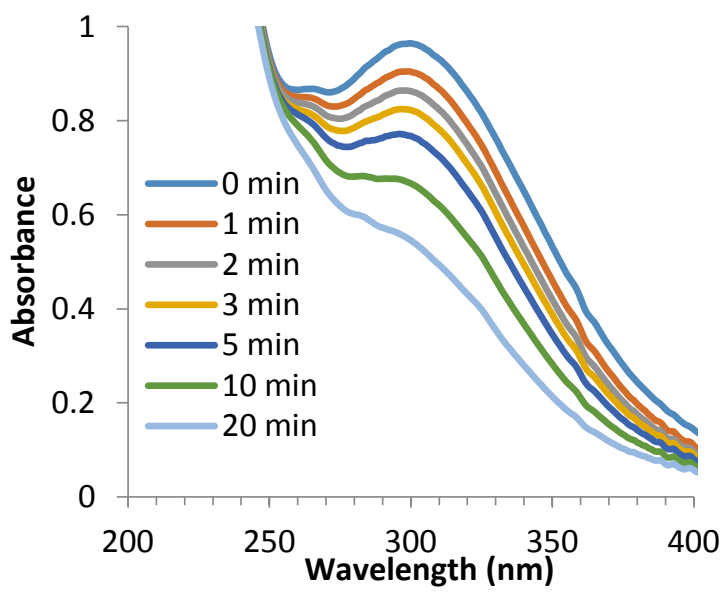

doping, the degradation of RO-diCN-PPV was much faster in the first 3 minutes of illumination and then slowed. Comparing the slopes of doped RO-diCN-PPV in the first 3 minutes and the undoped (Figure 4), the initial decay rate of doped sample was faster by $246 \%$.

\section{Characterization of Photodegradation Products}

To understand the different effects of DABCO, IR spectroscopy was used to examine components and functional groups evolution during the course of illumination.

C8-diCN-PPV. IR spectra of the undoped and doped films with different illumination time are in shown in Figure 5, and the following observations are made:

1) The integrated area of the $\mathrm{C}-\mathrm{H}$ peaks of the doped sample during 20 min of illumination remained constant, just as the undoped sample, consistent with a singlet oxygen mechanism.

2) The formation of $\mathrm{COOH}$ (with $\mathrm{C}=\mathrm{O}$ at 1716 and $\mathrm{OH}$ at $3425 \mathrm{~cm}^{-1}$ ) and oxo-acetonitrile $\left[\mathrm{C}(\mathrm{O}) \mathrm{CN}\right.$ with $\mathrm{C} \equiv \mathrm{N}$ at 2244 and $\mathrm{C}=\mathrm{O}$ at $1768 \mathrm{~cm}^{-}$ ${ }^{1}$, a product of ${ }^{1} \mathrm{O}_{2}$ cleavage of the dicyanovinylene and the precursor of $\mathrm{COOH}^{13}$ ] and the loss of the vinylenyl CN $\left(2223 \mathrm{~cm}^{-1}\right)$ are all slower in the

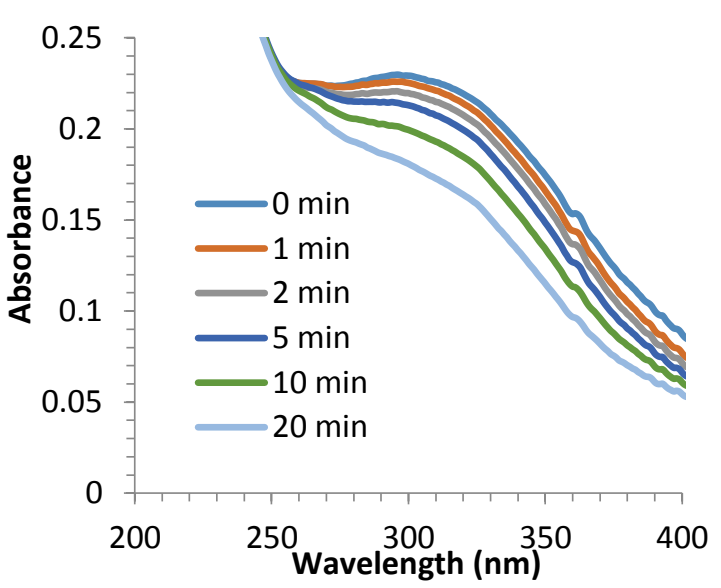

Figure 2. Evolution of UV-vis spectra of C8-diCN-PPV film (left) and C8-diCN-PPV film doped with 15 mol\% DABCO with illumination time. DABCO has some absorption at $260 \mathrm{~nm}$, which causes the polymer peak at $300 \mathrm{~nm}$ in the doped film to appear weaker. 

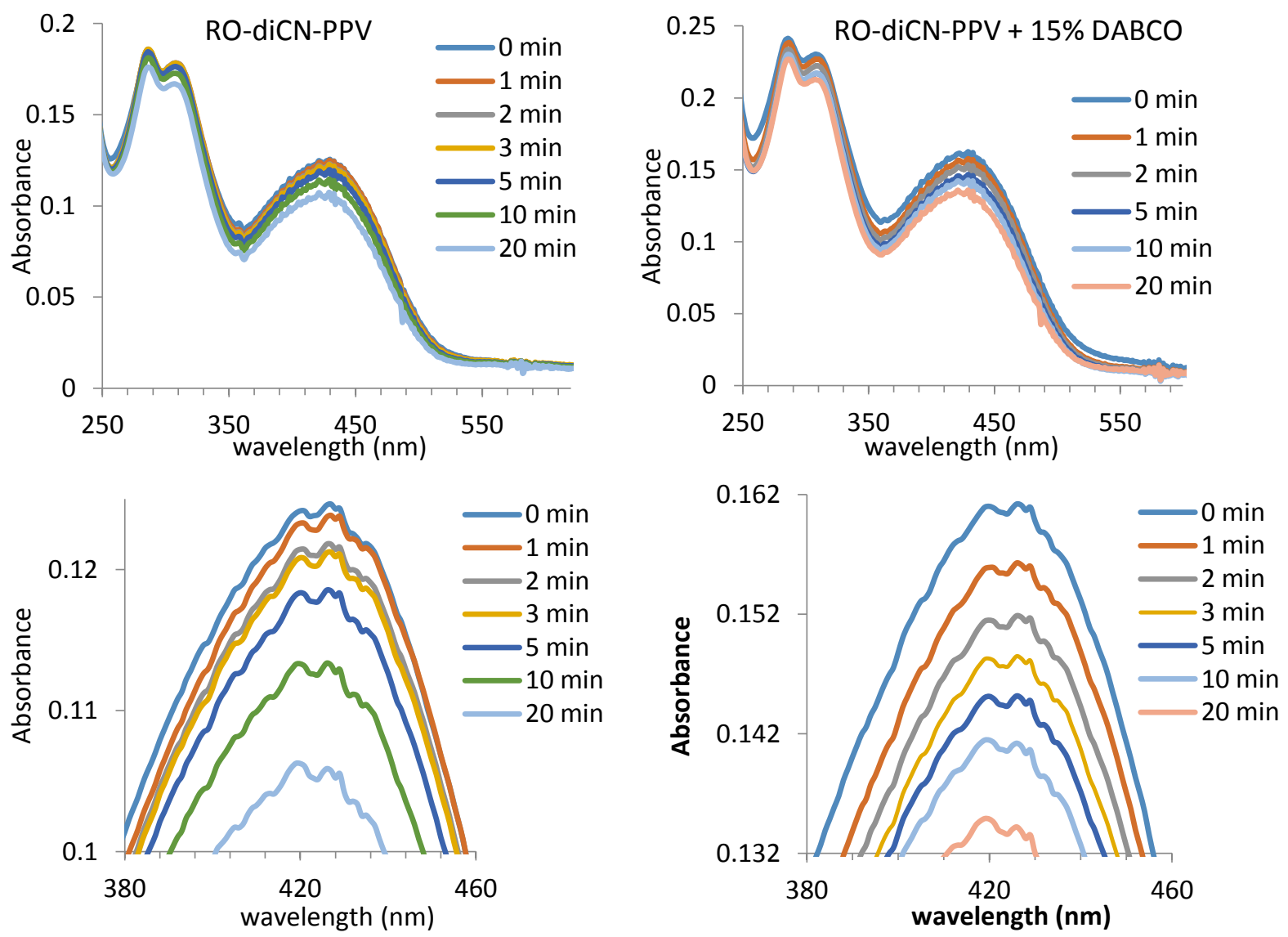

Figure 3. UV-vis spectra of RO-diCN-PPV film (top left) and 15 mol\% DABCO-doped RO-diCN-PPV film (top right) with increasing illumination time. Bottom are expanded views of the top plots to show that degradation is significantly faster in the doped film in the first few minutes.

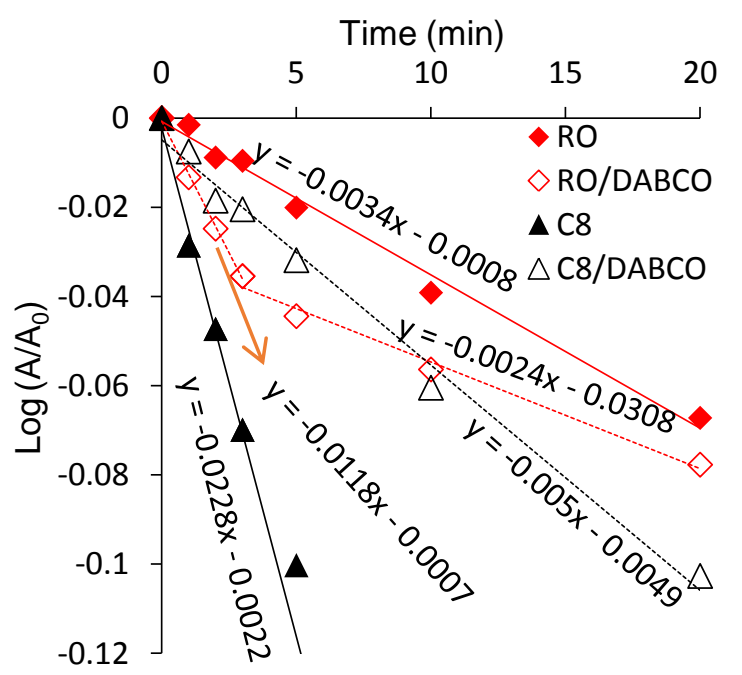

Figure 4. Semilog plots of normalized peak absorbance vs. illumination time. doped sample (Figure 5B) in agreement with the slower degradation observed in the UV-vis measurements. This further supports the singlet oxygen mechanism because DABCO is a singlet oxygen quencher and there is no report on its ability to inhibit a radical process.

3) The DABCO in the doped sample was not consumed during the 20 minutes of illumination, in stark contrast to the fate of DABCO in RO-diCN-PPV. This observation also helps exclude the involvement of a radical process as it is known that DABCO reacts with oxygen and other free radicals. ${ }^{20}$

4) The $\mathrm{C}(\mathrm{O}) \mathrm{CN}$ carbonyl peak $\left(1768 \mathrm{~cm}^{-1}\right)$ is as strong as the $\mathrm{COOH}$ carbonyl peak $\left(1716 \mathrm{~cm}^{-1}\right)$ in both samples, while the same signal in the earlier report ${ }^{13}$ of the undoped sample only 
appeared as a shoulder of the $\mathrm{COOH}$ carbonyl peak. This is because $\mathrm{C}(\mathrm{O}) \mathrm{CN}$ is not stable in film and IR measurements in this study were performed with less delay after illumination $(<1$ minute opposed to 5-10 minutes). Previously, it was noted that $\mathrm{C}(\mathrm{O}) \mathrm{CN}$ hydrolyzed completely when the illuminated films were transferred from slides to $\mathrm{KBr}$ plates using a solvent $\left(\mathrm{CH}_{2} \mathrm{Cl}_{2}\right) \cdot{ }^{13}$
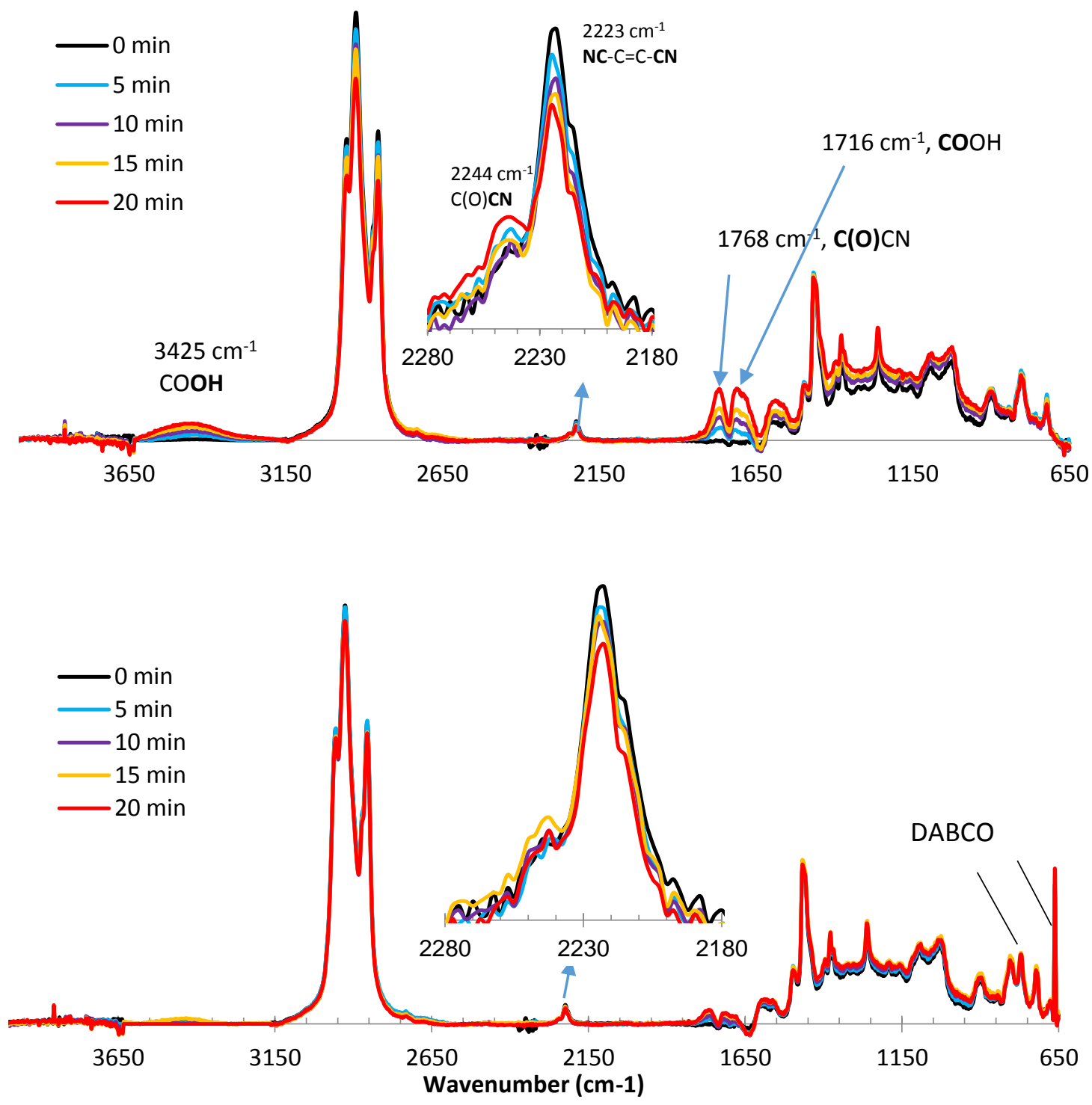

Figure 5. IR spectra of C8-diCN-PPV without (Top) and with (Bottom) 15 mol\% DABCO illuminated for 0, $5,10,15$ and 20 minutes.

RO-diCN-PPV. The IR spectra of the pristine and 15 mol\% DABCO-doped polymer samples with different illumination time are shown in Figure 6. Hardly any change happened to the undoped
RO-diCN-PPV, showing again the excellent photostability of this polymer. ${ }^{13}$

The following changes happened to the doped sample after illumination: 
1) $80 \%$ loss of DABCO after 5 minutes of the illumination and complete loss at 20 minutes.

2) A notable loss (3\%) in $\mathrm{C}-\mathrm{H}$ in $2350-3100 \mathrm{~cm}^{-1}$ and a gain in $\mathrm{O}-\mathrm{H}$ in $3100-3600 \mathrm{~cm}^{-1}$ after the first 5 minutes. In the next 15 minutes, only $1 \%$ degradation of $\mathrm{C}-\mathrm{H}$ was observed. The $\mathrm{OH}$ peak was not from $\mathrm{COOH}$ since no peak rose in the carbonyl region (in other words, $\mathrm{COOH}$ was not produced).

3) Partial loss of the original $\mathrm{CN}$ peak intensity and rise of a new $\mathrm{CN}$ peak at $2243 \mathrm{~cm}^{-1}$ mostly in the initial 5 minutes (Inset of Figure 6 bottom). The new $\mathrm{CN}$ peak, which is not from oxo-acetonitrile as no peak rises in the carbonyl region, is assigned to product $\mathbf{Q}$ in Scheme 2, which has two cyano-containing moieties: cyanohydrin and phenylacetonitrile. The peak position is close to those of naththalenyl cyanohydrin $\left(2248 \quad \mathrm{~cm}^{-1}\right)^{21}$ and phenylacetonitrile $\left(2252 \mathrm{~cm}^{-1}\right)$. From the relative peak intensities of vinylenyl cyano $\left(2216 \mathrm{~cm}^{-1}\right)$ and the cyano groups of $\mathbf{M}(2243$ $\mathrm{cm}^{-1}$ ) (in the inset of Figure 6), the amount of decomposed vinylene units is estimated to be $26 \%$ (or on average 4 units per polymer chain) at 5 minutes. The change after 5 min was much slower.

Are phenolic products generated in DABCOdoped RO-diCN-PPV? In photodegradation of RO-PPV, both carboxylic acid and phenolic products are produced and give a broad peak around $3400 \mathrm{~cm}^{-1}$. For DABCO-doped RO-diCNPPV, carboxylic acid products have been ruled out. To help determine the presence of phenolic products in DABCO-doped RO-diCNPPV, a 15 mol\% p-methoxyphenol doped ROdiCN-PPV sample was studied by IR spectroscopy. Three peaks involving phenolic $\mathrm{OH}$ are identified at 3420,1511, and $1227.5 \mathrm{~cm}^{-}$ 1 and are labeled on the spectrum (Figure 7). The $1227.5 \mathrm{~cm}^{-1}$ peak is

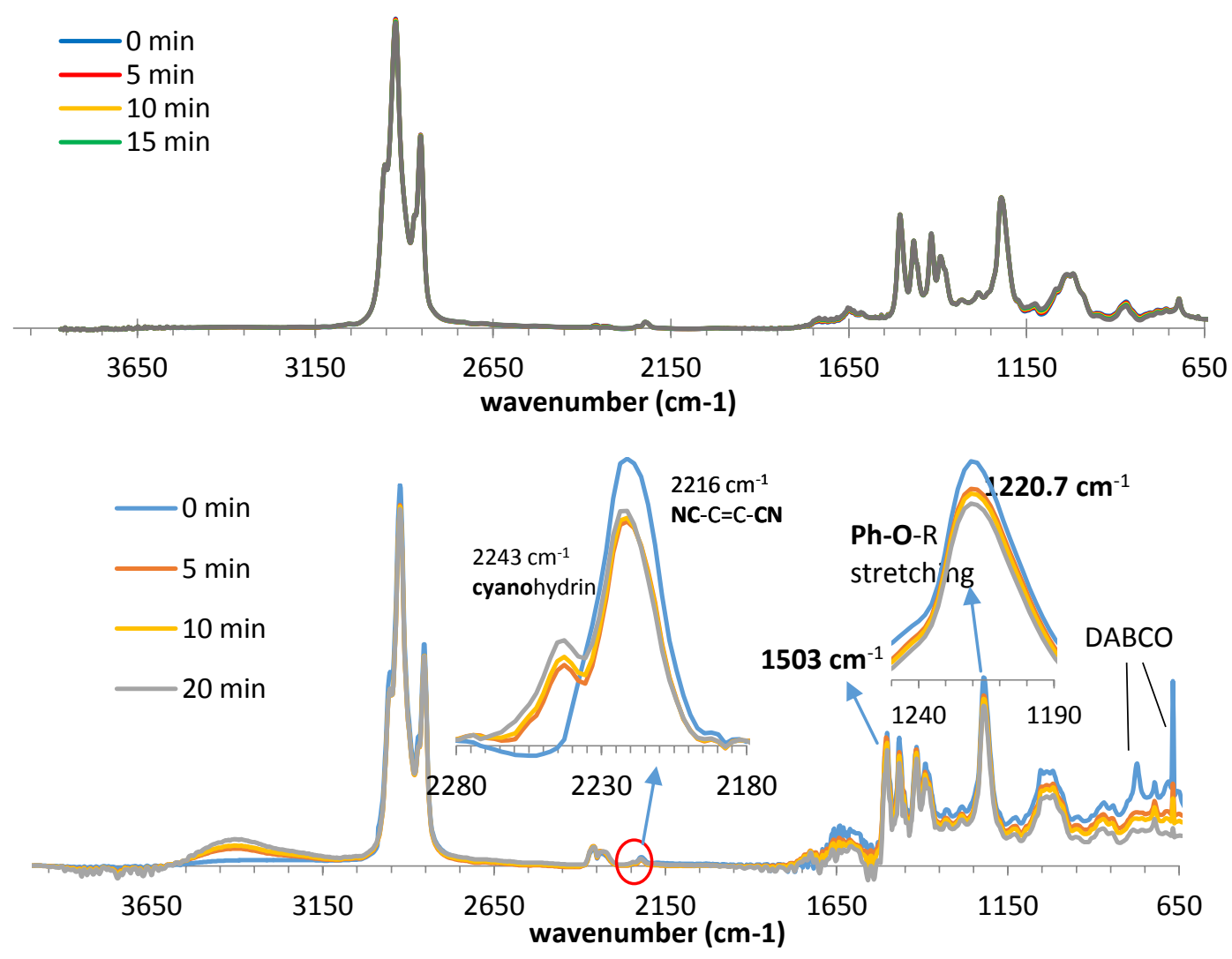


Figure 6. IR spectra of RO-diCN-PPV without (Top) and with (Bottom) $15 \mathrm{~mol} \%$ DABCO illuminated for different time. The apparent stronger signal of 0 min sample around $1650 \mathrm{~cm}^{-1}$ is due to the more tilted baseline.

- p-methoxyphenol doped RO-diCN-PPV

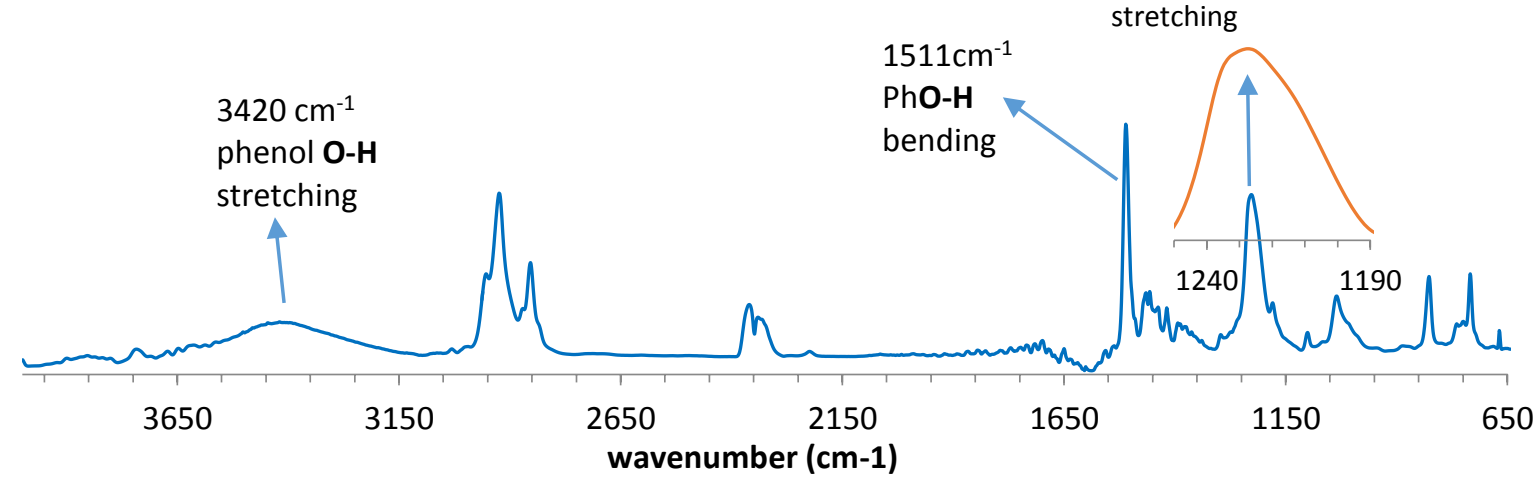

Figure 7. IR spectrum of 15 mol\% p-methoxyphenol doped RO-diCN-PPV, showing that phenolic $\mathrm{OH}$ is involved in three peaks at 3420,1511, and $1231 \mathrm{~cm}^{-1}$. The $1231 \mathrm{~cm}^{-1}$ peak overlaps with the stretching peak of Ph-OR of RO-diCN-PPV $\left(1220.7 \mathrm{~cm}^{-1}\right)$ to give a broad peak at $1227.5 \mathrm{~cm}^{-1}$.

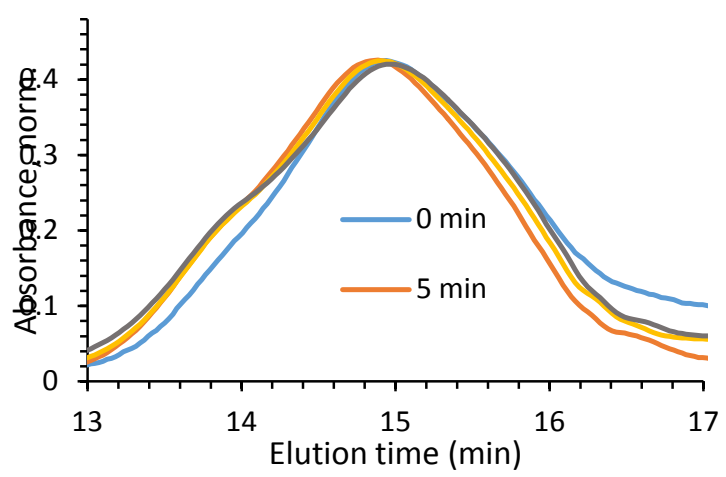

Figure 8. GPC elugram of $15 \mathrm{~mol} \%$ DABCOdoped RO-diCN-PPV illuminated for different time. Wavelength of detector: $310 \mathrm{~nm}$. The right-hand high tail of 0 min elugram was not from the polymer as the tail did not show up in the GPC curve monitored at $440 \mathrm{~nm}$.

an overlap of $\mathrm{C}-\mathrm{O}$ stretching of $\mathrm{Ph}-\mathrm{OH}(1231$ $\mathrm{cm}^{-1}$ ) of the dopant ${ }^{22}$ and Ph-OR of RO-diCNPPV (1220.7 $\mathrm{cm}^{-1}$, inset in Figure 6 bottom). In the IR spectra of DABCO-doped RO-diCN-PPV (Figure 6 bottom), no indications of phenolic $\mathrm{OH}$, i.e., intensity increase at/near $1511 \mathrm{~cm}^{-1}$ and broadening/shift of the $1220.7 \mathrm{~cm}^{-1}$ peak, are found. So, phenolic products were not produced in photodegradation of DABCO-doped RO-diCN-PPV.

GPC study of DABCO-doped RO-diCN-PPV. Changes in GPC elugrams with illumination time are notable when the plots are zoomed into the peak area (Figure 8Figure 7). First, after 5 min illumination a shoulder peak appeared on the left slope at the elution time of 13.78 minutes, corresponding to a peak MW $\left(M_{p}\right)$ of 29,000 which is 3.7 times the $M_{p}$ of the pristine ROdiCN-PPV, and the shoulder peak stayed after further exposure to light. Second, the peak retention time decreased from 14.93 minutes $\left(M_{p}=7,800\right)$ for the fresh sample $(0 \mathrm{~min})$ to 14.88 minutes $\left(M_{p}=8,400\right)$ for the $5 \mathrm{~min}$ sample. Further illumination caused the retention time to increase to 14.90 minutes $\left(M_{p}\right.$ $=8,100)$ for the 10 minutes sample and 14.97 minutes $\left(M_{p}=7,500\right)$ for the 20 minutes sample. These observations clearly indicate that a radical process, which increases $\mathrm{MW}$ by radical combination and radical addition, happened in the first five minutes, and yielded the dominance to a singlet oxygen mechanism, 
which reduced $\mathrm{MW}$ by cleaving $\mathrm{C}=\mathrm{C}$ bond, ${ }^{13}$ when DABCO was mostly consumed.

\section{Radical photodegradation mechanism of DABCO-doped RO-diCN-PPV}

The above observations of DABCO-doped ROdiCN-PPV indicate that a radical process was introduced by $D A B C O$ and dominated the photodegradation before DABCO was mostly consumed. To illustrate what happened between diCN-PPVs and DABCO upon illumination, molecular frontier orbitals energy $^{13}$ diagram, and photoexcitation and electron transfer processes are shown in Figure 9. With a HOMO level $(-6.04 \mathrm{eV})$, lower than that of DABCO $\left(-4.9 \mathrm{eV}^{23}\right.$, or $-5.07 \mathrm{eV}$ from our CV measurement), RO-diCN-PPV can oxidize DABCO into a radical cation upon photoexcitation. $^{24,23}$ Photoinduced electron transfer (PET) to other molecules such as carbonyl compounds to form reactive radical cations, ${ }^{25,26,27}$ has been documented for tertiary amines. Amine radical cations are very reactive ${ }^{23}$ and can degrade itself, the side chains and the vinylene units of RO-diCN-PPV via hydrogen abstraction, radical addition, and $\alpha$ cleavage. ${ }^{25,26}$ Relative to other tertiary amine radical cations, DABCO radical cation is significantly more stable and less acidic. ${ }^{28,29}$ Nevertheless, it was shown that it can undergo acid-base reaction with a neutral DABCO and get converted into a carbon radical in the time scale of seconds in solutions (Scheme 2 ). ${ }^{24}$

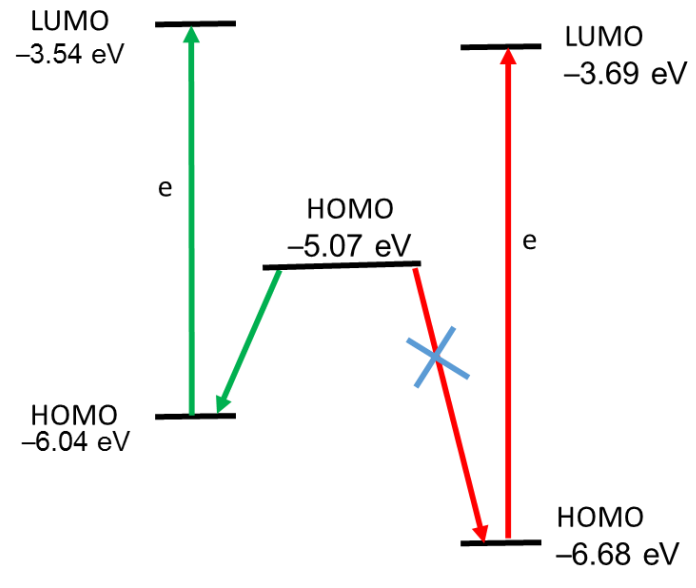

RO-diCN-PPV DABCO C8-diCN-PPV

Figure 9. Energy diagram of diCN-PPVs and DABCO. Upon photoexcitation, an electron is promoted into the LUMO, leaving a hole in HOMO. An electron in the HOMO of DABCO can transfer efficiently to the hole of RO-diCN-PPV, but not to the hole of C8-diCN-PPV.

Apparently, the energy difference between the HOMO of C8-DiCN-PPV $(-6.68 \mathrm{eV})^{13}$ and the HOMO of DABCO is too large for an efficient electron transfer from DABCO HOMO to the polymer HOMO to occur. Therefore, DABCO doping does not introduce a radical process to C8-DiCN-PPV.

Radical reactions of the side chain in DABCOdoped RO-diCN-PPV. Schemes 1 shows radical reactions of the alkoxy side chain, which is partially adapted from the literature. ${ }^{7}$ The position $\alpha$ to the oxygen atom is well-known for its high reactivity. ${ }^{7,30}$ A hydrogen on an $\alpha$ carbon may be abstracted by a DABCO cationic radical or an oxygen radical generated during the radical process (Scheme 2) to form a carbon radical $\mathbf{B}$. Two possible reactions may happen to B: addition to a vinylene unit (the blue path in Scheme 1) and/or reaction with $\mathrm{O}_{2}$ to form a peroxy radical $\mathbf{C}$ (the red path adapted from that for RO-PPV). ${ }^{7}$ The red path would led to production of ester $\mathbf{F}\left(\sim 1760 \mathrm{~cm}^{-1}\right)$, formate $\mathbf{G}$ $\left(1740 \mathrm{~cm}^{-1}\right)$, phenol $\mathbf{I}$, and aliphatic acids ( 1732 $\left.\mathrm{cm}^{-1}\right)$. However, no evidences of their presence are found in the IR spectra of DABCO-doped ROdiCN-PPV (Figure 6 bottom). So, the red path is 
not active for RO-diCN-PPV. This is unexpected but justifiable because the dicyanovinylene is a much stronger radical receptor than the vinylene in RO-PPV as electron withdrawing groups can greatly increase the reactivity of alkenes for radical addition. ${ }^{31}$ For example, a cyano substitution increases radical addition reaction rate of ethylene by 174 times. Radical B may add to vinylene intra- or intermolecularly. The intermolecular addition was at least partially responsible for the increase in polymer MW.

Radical reactions of the backbone. In DABCOdoped RO-diCN-PPV, loss of absorbance is due to attack of radicals at the vinylene units. The mechanism outlined in Scheme 2 is adapted from that proposed by Chambon et al. for ROPPVs. ${ }^{6,8}$ The DABCO cationic radical (a) is acidic and can be deprotonated by a DABCO to form a carbon radical $\mathbf{c}^{24}$ The radical process could be initiated by different radicals including a, B, c, and possibly other radicals that were generated during the radical process. The rest of the pathway is different from that of RO-PPV ${ }^{6,8}$ in two ways: 1 ) the radical intermediate $\mathbf{N}$ could not do a cage reaction, ${ }^{8}$ which is favored reaction if a hydrogen is present instead of a $\mathrm{CN}$, and 2) $\mathrm{N}$ did not undergo $\beta$-scission (cleavage of the red colored bond in $\mathbf{N}, a$ significant way of evolution of alkoxy radicals ${ }^{7}$ ) since no carbonyl peak (from P) was observed. Radical $\mathbf{N}$ abstracts a hydrogen from a hydrogen source to form the cyanohydrin $\mathbf{Q}$ which is quite stable. $^{32}$ The $\mathrm{OH}$ in cyanohydrin is responsible for the broad OH peak (3100-3600 cm $\mathrm{cm}^{-1}$ ) (Note: $\mathrm{OH}$ in naphthalenyl cyanohydrin peak at peak near $\left.3418 \mathrm{~cm}^{-1}\right){ }^{21}$

Scheme 1. Radical reactions of the alkoxy side chains of RO-diCN-PPV doped with DABCO. $R^{\prime} \bullet=$ DABCO radical cation or an oxygen radical.

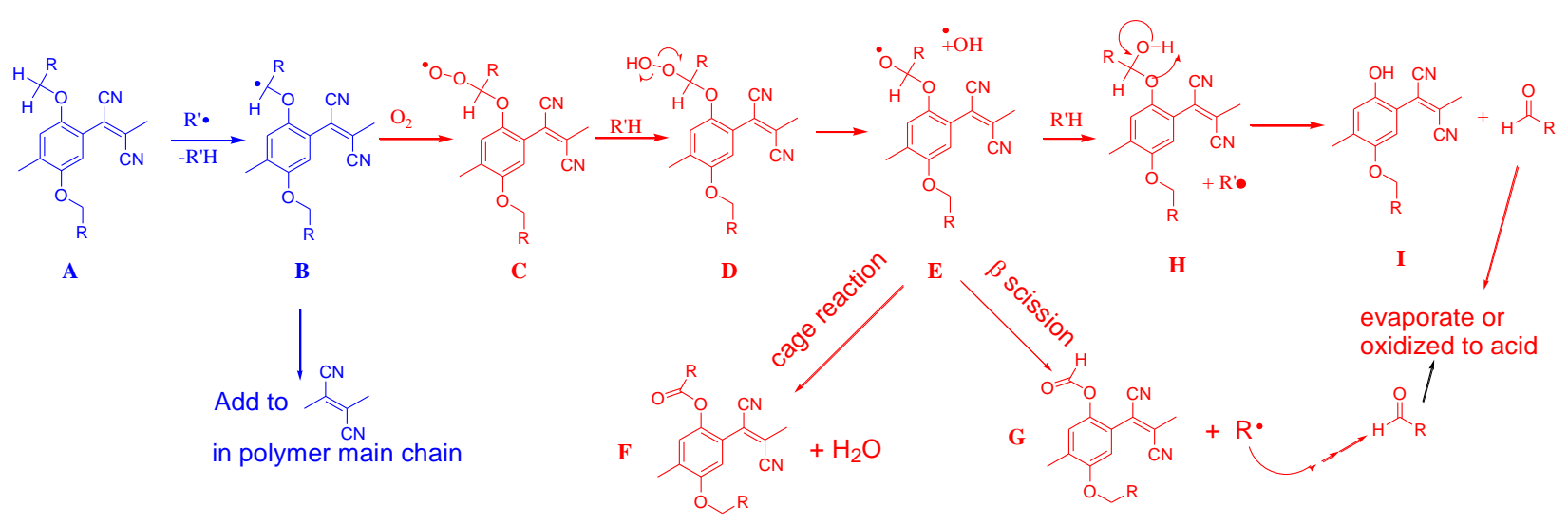

The PET between RO-diCN-PPV and DABCO also produced polymer radical anions which is similar to $\mathbf{K}$ and may follow the same path in
Scheme 2 to eventually give a product similar to Q. The anion can obtain a proton from a DABCO cationic radical (a) to turn into a neutral radical.

Scheme 2. Radical degradation of vinylene backbone in RO-DiCN-PPV initiated by the radical cation of DABCO. $R^{\prime} \bullet=D A B C O$ radical cation $(\mathbf{a}), \mathbf{B}, \mathbf{c}$, or other radicals that were generated during the radical process. 


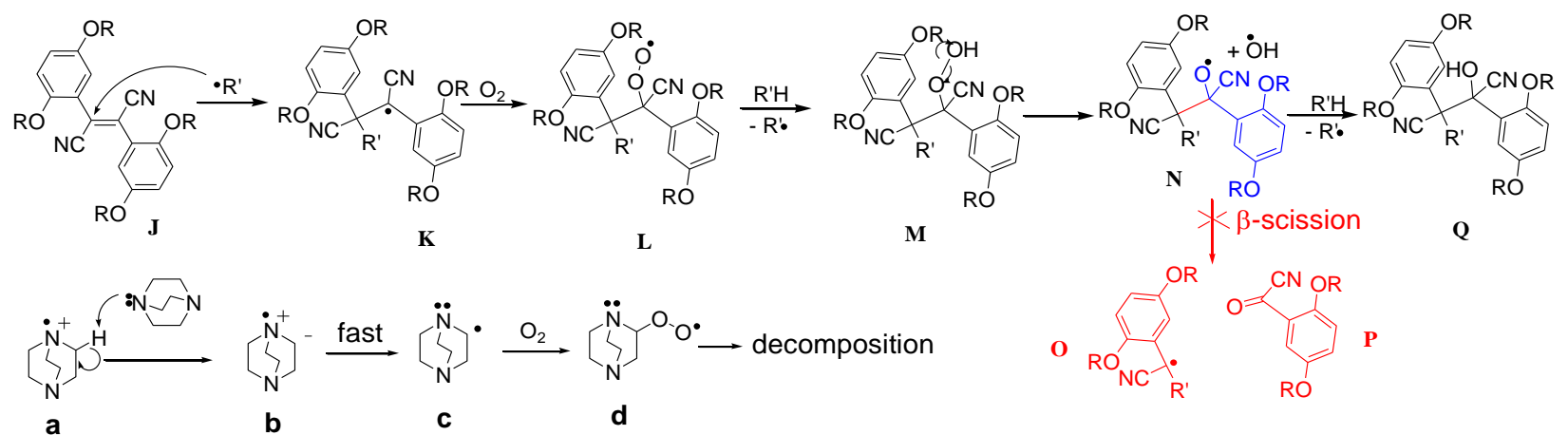

\section{CONCLUSIONS}

The effects of DABCO on the photostability of RO-diCN-PPV and C8-diCN-PPV were investigated. To our surprise, the singlet oxygen quencher had opposite effects on photodegradation of these two polymers. With 15 mol\% DABCO doping, degradation rate of C8diCN-PPV film decreased by $65 \%$, while the degradation rate of RO-diCN-PPV increased by $246 \%$ in the first 3 minutes of exposure to intense white light and then slowed, as indicated by UV-vis absorption. IR experiments showed that DABCO content in C8-diCN-PPV film remained unchanged during 20 minutes of illumination, but lost $80 \%$ in RO-diCN-PPV in only 5 minutes. With the loss of the most of $\mathrm{DABCO}$, degradation of vinylene units and loss of $\mathrm{CH}$ immediately slowed down.

All the IR and GPC MW analysis results suggest that DABCO slowed down degradation of C8diCN-PPV without altering the degradation mechanism, but accelerated RO-diCN-PPV photodegradation and switched the dominant mechanism from singlet oxygen to radical oxygen. The difference arises from the difference in their side chains. The alkoxy in ROdiCN-PPV donates electron and raise the HOMO energy from $-6.68 \mathrm{eV}$ for C8-diCN-PPV to -6.04 $\mathrm{eV}$, which is still lower than the HOMO of DABCO, but not too low to shut down electron transfer from the HOMO of DABCO to the HOMO of RO-diCN-PPV where holes are generated upon photoexcitation. The result of IR product analysis indicates that the side chain carbon radicals did not follow the usual path (i.e., reacting with $\mathrm{O}_{2}$ and eventually getting cleaved), but rather added to the dicyano substituted $\mathrm{C}=\mathrm{C}$ bonds. One consequence of the deviation(s) from the side chain radical decomposition of RO-PPV is that the broad $\mathrm{OH}$ IR peak is now assigned to cyanohydrin - a decomposition product of dicyanovinylene, rather than phenols and carboxylic acids that are generally produced in radical decomposition of RO-PPVs. Mechanisms have been modified to rationalize the observed differences from radical decomposition of RO-PPV.

The study presents an interesting case where addition of a singlet oxygen quencher produces opposite effects on photodegradaton of two polymers that are only different in side chain.

\section{ACKNOWLEDGEMENTS}

This material is based upon work supported by the U.S. Air Force Office of Scientific Research under Award No. FA955010-1-0555 and South Dakota NSF EPSCoR Program (Grant No. 0903804). LS is grateful for the funding from NSF SD IGERT at U. South Dakota (Grant No. 0903685)

\section{REFERENCES AND NOTES}

1. Manceau, M.; Bundgaard, E.; Carlé, J. E.; Hagemann, O.; Helgesen, M.; Søndergaard, R.; Jørgensen, M.; Krebs, F. C., J. Mater. Chem. 2011, 21, 4132-4141.

2. Tromholt, T.; Madsen, M. V.; Carlé, J. E.; Helgesen, M.; Krebs, F. C., J. Mater. Chem. 2012, 22, 7592-7601. 
3. Kutuvantavida, Y.; Williams, G. V. M.; Bhuiyan, M. D. H.; Raymond, S. G.; Kay, A. J., J. Phys. Chem. C 2015, 119, 3273-3278.

4. Foucher, C.; Guilhabert, B.; Herrnsdorf, J.; Laurand, N.; Dawson, M. D., Optics Express 2014, 22, 24160-24168.

5. Moretti, E.; Talon, A.; Storaro, L.; Donne, A. L.; Binetti, S.; Benedetti, A.; Polizzi, S., J. Lum. 2014, 146, 178-185.

6. Chambon, S.; Rivaton, A.; Gardette, J. L.; Firon, M., Polym. Degrad. Stab. 2011, 96, 1149-1158.

7. Chambon, S.; Rivaton, A.; Gardette, J. L.; Firon, M.; Lutsen, L., Journal of Polymer Science Part A: Polymer Chemistry 2007, 45, 317-331.

8. Chambon, S.; Rivaton, A.; Gardette, J. L.; Firon, M., Journal of Polymer Science Part A: Polymer Chemistry 2009, 47, 6044-6052.

9. Manceau, M.; Rivaton, A.; Gardette, J. L.; Guillerez, S.; Lemaitre, N., Polym. Degrad. Stab. 2009, 94, 898-907.

10. Hoke, E. T.; Sachs-Quintana, I. T.; Lloyd, M. T.; Kauvar, I.; Mateker, W. R.; Nardes, A. M.; Peters, C. H.; Kopidakis, N.; McGehee, M. D., Adv. Energy Mater. 2012, (2), 13511357.

11. Dam, N.; Scurlock, D.; Wang, B.; Ma, L.; Sundahl, M.; Ogilby, P. R., Chem. Mater. 1999, 11, 1302-1305.

12. Sun, J.; Sanow, L. P.; Sun, S. S.; Zhang, C., Macromolecules 2013, 46, 4247-4254.

13. Sanow, L. P.; Sun, J.; Zhang, C., J. Polym. Sci. A Polym. Chem. 2015.

14. Moura, J. C. V. P.; Oliveira-Campos, A. M. F.; Griffiths, J., Dyes and Pigments 1997, 33, 173-196.

15. Tian, Z.; Yu, J.; Wang, X.; Groff, L. C.; Grimland, J. L., J. Phys. Chem. B 2013, 117, 4517-4520.

16. Ouannes, C.; Wilson, T., J. Am. Chem. Soc. 1968, 90, 6527-6528.

17. Steiner, U.; Winter, G.; Kramer, H. E. A., J. Phys. Chem. 1977, 81, 1104-1110.
18. Valnes, K.; Brandtzaeg, P., J. Histochem. Cytochem. 1985, 33, 755-761.

19. Ogryzlo, E. A.; Tang, C. W., J. Am. Chem. Soc. 1970, 92, 5034-5036.

20. Packer, J. E.; Mahood, J. S.; Mora-Arellano, V. O.; Slater, T. F.; Willson, R. L.; Wolfenden, B. S., Biochem. Biophys. Res. Commun. 1981, 98, 901-906.

21. Yosef, H. A. A.; Morsy, N. M.; Mahran, M. R. H.; Aboul-Enein, H. Y., J. Iran. Chem. Soc. 2007, 4, 46-58.

22. Silverstein, R. M.; Webster, A. X.; Kiemle, D. J., Spectrometric Identification of Organic Compounds. 7th ed.; John Wiley \& Sons, Inc.: 2005.

23. Vasudevan, D.; Wendt, H., J. Electroanal. Chem. 1995, 192, 69-74.

24. Zheng, Z.-R.; Evans, D. H.; Nelsen, S. F., J. Org. Chem. 2000, 65, 1793-1798.

25. Hasegawa, E.; Tosaka, E.; Yoneoka, A.; Tamura, Y.; Takizawa, S.; Tomura, M.; Yamashita, Y., Radical Cations Res. Chem. Intermed. 2013, 39, 247-267.

26. Hasegawa, E.; Ishiyama, K.; Fujita, T.; Kato, T.; Abe, T., J. Org. Chem. 1997, 62, 23962400.

27. Kaise, M.; Someno, K., Chem. Lett. 1987, 1, 1295-1298.

28. Chow, Y. L.; Danen, W. C.; Nelsen, S. F.; Rosenblatt, D. H., Chem. Rev. 1978, 78, 243274.

29. Dinnocenzo, J. P.; Banach, T. E., J. Am. Chem. Soc. 1989, 111, 8646-8653.

30. Gardette, J.-L.; Mailhot, B.; Posada, F.; Rivaton, A.; Wilhelm, C., Macromol. Symp. 1999, 143, 95-109.

31. Zytowski, T.; Fischer, H., J. Am. Chem. Soc. 1996, 118, 437-439

32. Fomunyam, R. T.; Adegbola, A. A.; Oke, O. L., Food Chem. 1985, 17, 221-225. 


\section{Opposite Effects of A Singlet Oxygen Quencher on Photochemical Degradation of Dicyano-Substituted Poly(phenylenevinylenes) with Different Side Chains}

Logan P. Sanow and Cheng Zhang

RO-diCN-PPV and C8-diCN-PPV photodegrade via singlet oxygen mechanism and RO-diCN-PPV is seven times more stable. 1,4-diazabicyclo[2.2.2] octane (DABCO), a well-known singlet oxygen quencher, exhibits opposite effects on photodegradation of the polymers. With 15 mol\% DABCO, degradation rate of C8-diCN-PPV decreased by $65 \%$, while that of RO-diCN-PPV increased by $246 \%$. Electron donation from DABCO to RO-diCN-PPV can happen upon photoexcitation and results in generation of DABCO cationic radicals which initiate a radical process that damages the polymer and destroys DABCO.

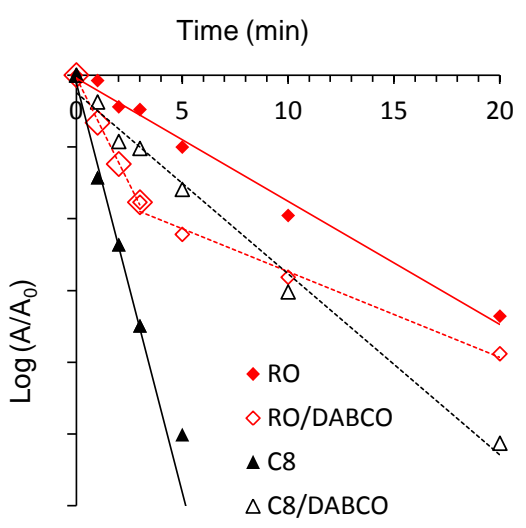

